\title{
User Study on Informationally Equivalent Thematic Maps for Map Use Tasks
}

\author{
Katarzyna Słomska-Przech ${ }^{\mathrm{a}, *}$, Izabela Małgorzata Gołębiowska ${ }^{\mathrm{a}}$ \\ ${ }^{a}$ University of Warsaw, Faculty of Geography and Regional Studies, Department of Geoinformatics, Cartography and \\ Remote Sensing - k.slomska@uw.edu.pl, i.golebiowska@uw.edu.pl \\ * Corresponding author
}

Keywords: thematic map; user study; map types; choropleth map; isoline map; graduated symbols map; task type

\begin{abstract}
:
Thematic maps, which can present the same quantitative data - choropleth map, dot map, isoline, proportional symbols, and graduated symbols - emphasize various aspects of mapped phenomena and therefore focus readers' attention on different issues. Depending on the way a map is designed, it can be recommended for different types of tasks. For example, isoline maps are recommended for presenting the magnitude, choropleth maps are advised for presenting overall geographical patterns of the mapped variable, whereas proportional and graduated symbols would be a good choice for comparing values, especially in neighboring fields (Dent et al. 2009, Slocum et al. 2010, Tyner 2010). This corresponds to the taxonomy proposed by MacEachren and DiBiase (1991) in which smooth (e.g. isoline) and abrupt (e.g. choropleth), as well as discrete (e.g. graduated symbols) and continuous (e.g. isoline) types of geovisualisation are juxtaposed.
\end{abstract}

However, recommendations from handbooks should be supported by empirical evidence. In the presented study, we compared three commonly used map types, namely choropleth, graduated symbols, and isoline (Figure 1), to evaluate how they differ in facilitating solving 11 analytical tasks of a different kind (identify, find extremum, distinguish, retrieve value, compare, interpret, categorize, cluster, sort, correlate, locate) (Roth 2012). Another issue investigated in the study was the consistency of performance metrics and subjective metrics, since previous studies on this matter indicate that users do not always prefer the solutions which result in the best performance metrics (Hegarty 2009).

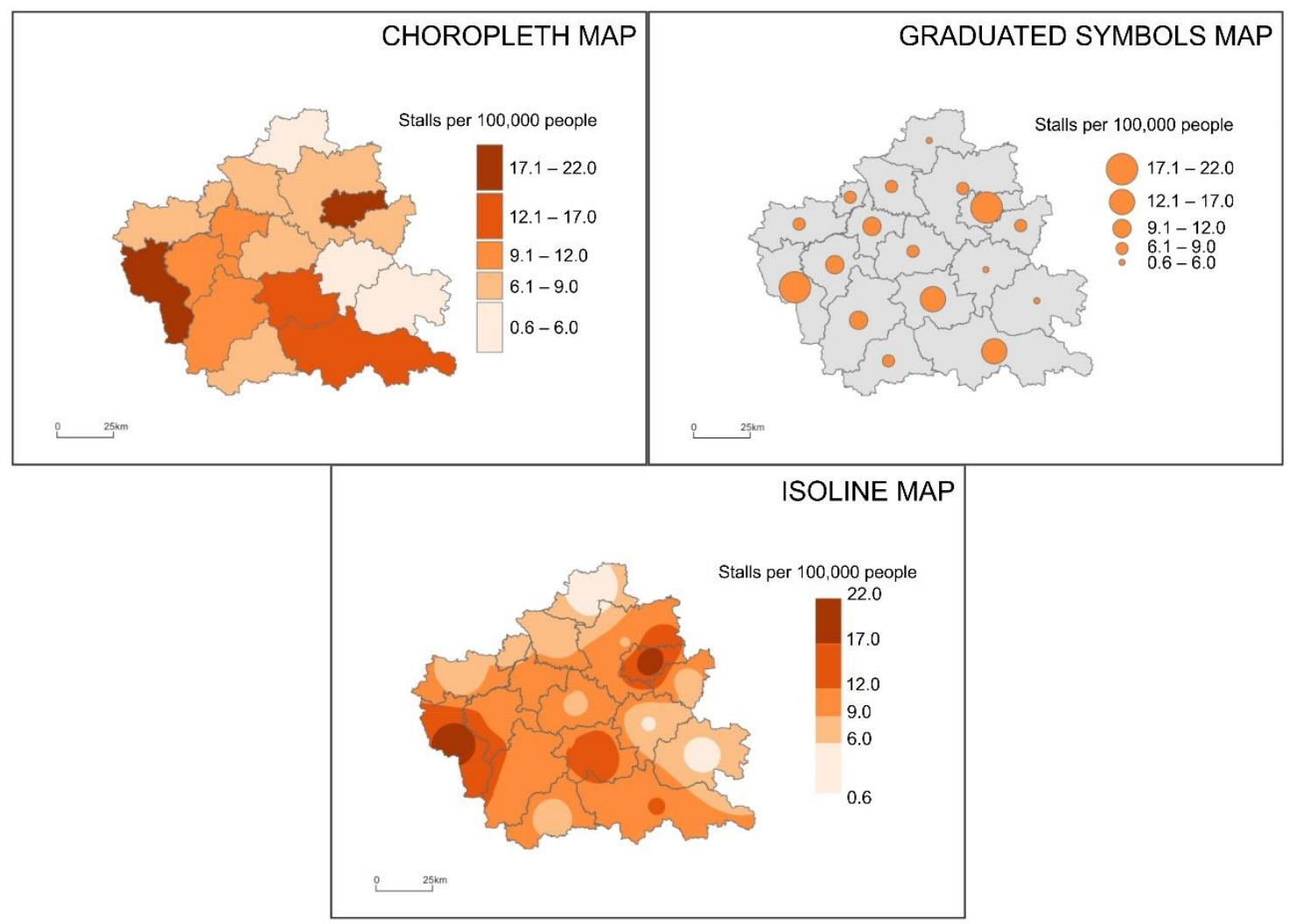

Figure 1. The tested map types: choropleth map, graduated symbols map, and isoline map. 
We conducted a user study in Poland with 366 high school students (15-20 years old). Each respondent answered the questions individually. The study was conducted with use of a web application. We compared three map types presenting the same input data (Figure 1). Three metrics were examined: two performance metrics - answer accuracy and time, and one subjective metric - the difficulty of the task.

The best results of all metrics (answer accuracy and time, the rated difficulty of the tasks) for all tasks were obtained by participants working with the choropleth map. Slightly worse general results were obtained by the respondents working with the graduated symbols map, and the worst while working with isolines map. Therefore, for the basic, analytical tasks applied in our study, the obtained results on performance are not coherent with recommendations from handbooks, which suggest that different tasks should favor different map types (Dent et al. 2009, Slocum et al. 2010, Tyner 2010). Interestingly, only in one case of the sorting task, the effectiveness of participants using isolines map was higher than users of graduated symbols map. What is more, the results on performance metrics (answer accuracy and time) were consistent with results on the subjective metric - rated difficulty of tasks.

The conducted study showed that a choropleth map could be the most efficient and effective map type for the tested tasks. Yet, we are aware of the limitations of this map type, e.g. ability to read values only for the whole area (enumeration unit), not referred to a specific point in space. We presume that the frequent training in choropleth maps in school education, as shown by the analysis of school atlases (Havelková, Hanus 2018), seems to result in a high level of literacy with regard to reading this map type. We believe that the richness of thematic cartography should not be abandoned, and training and further work should not be limited to one favorable map type only. Moreover, it should be noticed that our study focused only on basic, analytical tasks and did not refer to more complex tasks (e.g. problem-solving or decisionmaking). Therefore, studies comparing informationally equivalent maps should be continued.

\section{Acknowledgments}

This research was funded by the National Science Centre, Poland, grant number UMO-2016/23/B/HS6/03846, "Evaluation of cartographic presentation methods in the context of map perception and effectiveness of visual transmission".

\section{References}

Dent, B.D., Torguson, J.S., Hodler, T.W., 2009. Cartography, 6th ed.; McGraw-Hill Higher Education: Boston, MA, USA.

Havelková, L., Hanus, M., 2018, The Impact of Map Type on the Level of Student Map Skills. Cartogr. Int. J. Geogr. Inf. Geovisualization. 53, 3, pp. 149-170.

Hegarty, M., Smallman, H.S., Stull, A.T., Canham, M.S., 2009. Naïve Cartography: How Intuitions about Display Configuration Can Hurt Performance. Cartogr. Int. J. Geogr. Inf. Geovisualization 44, 3, pp. 171-186.

MacEachren, A. M., DiBiase, D., 1991. Animated maps of aggregate data: Conceptual and practical problems. Cartography and Geographic Information Systems, 18, 4, pp. 221-229.

Roth, R.E., 2012. Cartographic Interaction Primitives: Framework and Synthesis. Cartographic Journal, 49, $376-395$.

Slocum, T.A., McMaster, R.B., Kessler, F.C., Howard, H.H., 2010. Thematic Cartography and Geovisualization, 3rd ed.; Prentice Hall Series in Geographic Information Science; Pearson Prentice Hall: Upper Saddle River, NJ, USA.

Tyner, J.A. 2010. Principles of Map Design; Guilford Press: New York, NY, USA. 